Nervenarzt 2010 $\cdot 81: 795-795$ DOI 10.1007/s00115-010-3004-x

Online publiziert: 30. Juni 2010

(c) Springer-Verlag 2010

\author{
W. Maier · F. Jessen \\ Klinik und Poliklinik für Psychiatrie und Psychotherapie, Universitätsklinikum Bonn
}

\title{
Evidenzbasierte Standards für die Versorgung von Patienten mit Demenzen
}

\section{Die interdisziplinäre S3-Leitlinie „Demenzen"}

Dem stürmischen medizinischen Erkenntnisfortschritt in Diagnostik und Therapie von Demenzen stehen erhebliche Unzulänglichkeiten in der Versorgungspraxis gegenüber. Unsere diagnostischen und therapeutischen Möglichkeiten haben sich in den vergangenen 10 Jahren in diesem Bereich wesentlich weiterentwickelt und dies ist ebenso für die nahe Zukunft zu erwarten. Die Umsetzung des erreichten und durch klinische Studien bekräftigten Fortschritts in Diagnostik, Therapie und Betreuung in die Praxis ist bisher allerdings bestenfalls in Ansätzen gelungen. Es besteht eine deutliche Lücke zwischen dem evidenzbasierten Wissen um die besten Strategien und dem praktischen Handeln der Ärzte. Die Hindernisse für den Transfer wissenschaftlich belegter klinischer Strategien in die Praxis sind zahlreich und teilweise auch noch unbekannt. Eine wesentliche Voraussetzung für eine konsequente Nutzung wissenschaftlicher Erkenntnisse in der Versorgung sind aber die Ableitung und Verbreitung wissenschaftlich abgesicherter, praxisorientierter Standards für die medizinische Versorgung.

Solche Standards werden in Leitlinien niedergelegt, an denen sich das praktische ärztliche Handeln orientieren soll. Leitlinien sind um so wirksamer, je stärker evidenzbasiert sie sind und umso mehr sie für den Arzt nachvollziehbar und handlungsorientiert formuliert sind; sie haben eine umso stärkere Breitenwirkung, je umfänglicher der Konsens ist, auf den sie sich stützen können. Das von der Ar- beitsgemeinschaft wissenschaftlich-medizinischer Fachgesellschaften (AWMF) entwickelte Modell interdisziplinärer $\mathrm{S}_{3}$ Leitlinien verfolgt genau diese Ziele. S3Leitlinien bestehen aus Thesen mit Hintergrundtext, die das aktuell verfügbare, evidenzbasierte Wissen komprimieren und praxisorientiert darstellen; hinzu kommt die transparente Darlegung der Ergebnisse von Evidenzrecherchen und von Positionierungen anderer relevanter Leitlinien in einem Methodenbericht, der die angewandten Regeln und Verfahren nachvollziehbar darstellt.

Nach Abschluss des Konsensusprozesses ist seit November 2009 die interdisziplinäre $\mathrm{S}_{3}$-Leitlinie für Demenzen unter www.DGPPN.de und www.leitlinien.de zugänglich und befindet sich derzeit im Druck. Die beiden federführenden Fachgesellschaften DGPPN und DGN haben diesen Prozess zusammen mit der Alzheimer-Gesellschaft unter Mitwirkung der AWMF organisiert. 28 weitere wissenschaftliche Gesellschaften waren in der Experten- und in der Konsensusgruppe aktiv beteiligt. Wesentliche Komponenten dieser Leitlinie werden in vier Beiträgen in dieser Ausgabe von Der Nervenarzt dargestellt.

Die vorliegende $S_{3}$-Leitlinie stützt sich dabei teilweise auf primäre Evidenzrecherchen und auf Schlussfolgerungen aktueller Leitlinien anderer Länder, vor allem der Leitlinie des National Institute for Health and Clinical Excellence (www.nice.org.uk) und des Scottish Intercollegiate Guidelines
Network (www.sign.ac.uk). Die S3-Leitlinie ergänzt diese vorliegenden, international verbreiteten Leitlinien wesentlich, vor allem im Bereich der nichtmedikamentösen Therapieverfahren und der Behandlung von Verhaltenssymptomen bei Demenzen. Bereits früher wurden in Deutschland schon Leitlinien zu Demenzen entwickelt, z. B. eine S1-Leitlinie der DGPPN, eine S2-Leitlinie der DGN und eine S2E-Leitlinie der Deutschen Gesellschaft für Allgemeinmedizin (DEGAM). Aufgrund von Defiziten im methodischen Standard von Evidenzrecherchen oder in der Konsentierung der Leitlinienempfehlungen konnte keine dieser deutschsprachigen Leitlinien den von der AWMF definierten $S_{3}$-Status erreichen. Somit kann die nun vorliegende $\mathrm{S}_{3}$-Leitlinie „Demenzen“ eine hohe Verbindlichkeit für die Versorgungspraxis beanspruchen.

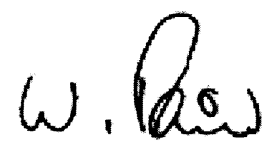

W. Maier

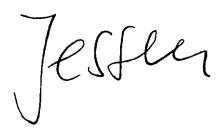

F. Jessen

\section{Korrespondenzadresse \\ Prof. Dr. W. Maier}

Klinik und Poliklinik für Psychiatrie und Psychotherapie, Universitätsklinikum Bonn Sigmund-Freud-Straße 25, 53105 Bonn Wolfgang.Maier@ukb.uni-bonn.de 